\title{
SÍNDROME DE BLACKFAN-DIAMOND: UM RELATO DE CASO
}

\section{BLACKFAN-DIAMOND SYNDROME: A CASE STUDY}

\begin{abstract}
Maria Vitória Moscardi Spack ${ }^{1 *}$, Juliano Smaniotto Silveira', Reginaldo José Andrade $^{2}$

${ }^{1}$ Acadêmica do nono período do curso de Medicina da Faculdade Assis Gurgacz. ${ }^{2}$ Médico formado pela Universidade Federal do Paraná, Oncologista clínico e Hematologista, mestre em Medicina Interna e docente da Universidade Estadual do Oeste do Paraná e do Centro Universitário Assis
\end{abstract} Gurgacz.

*Autor correspondente: mvitoriaspack@hotmail.com https://orcid.org/0000-0002-2252-7132

\section{RESUMO}

Introdução: A síndrome de Blackfan-Diamond é uma rara anemia congênita, diagnosticada no início da infância. Os principais sintomas apresentados são anemia, anormalidades ósseas e dificuldade de ganhar peso. O diagnóstico é obtido por hemograma e aspirado de Medula Óssea. O tratamento consiste em corticoterapia, programa de transfusão sanguínea e transplante de medula óssea. Relato do caso: Paciente, sexo masculino, 11 anos de idade, portador da síndrome com diagnóstico no primeiro mês de vida e refratário à corticoterapia, realiza transfusão sanguínea a cada 21 dias e espera por doador para transplante de medula óssea. Discussão: 0 paciente em questão apresenta a maioria dos sintomas clássicos da doença, porém não é responsivo a um dos principais tratamentos. Conclusão: Apesar da refratariedade ao tratamento com corticoterapia, o paciente apresenta-se estável no programa de transfusão enquanto aguarda doador compatível para transplante de medula óssea.

Palavras-chave: Síndrome. Blackfan-Diamond. Anemia. Transfusão.

\section{ABSTRACT}

Introduction: The Blackfan-Diamond Syndrome is a rare congenital anemia, diagnosed during the early childhood. The main symptoms are anemia, bone abnormalities and difficulties to gain weight. The diagnosis is based on the hemogram and bone marrow aspiration. The treatment consists in corticosteroid, blood transfusion program and bone marrow transplant. Case Study: Patient, male, 11 years old, carrier of the syndrome and diagnosed during his first month of life and refractive to the treatment with corticosteroid, performs a blood transfusion each 21 days and waits for a donor to perform a bone marrow transplant. Discussion: The said patient presents the majority of the classical symptoms of the disease, however, he is not responsive to one of the main treatments. Conclusion: Even though the refractivity to the treatment with corticosteroid, the patient presents himself stable at the blood transfusion program while waits for a compatible donor for the bone marrow transplant. Keywords: Syndrome. Diamond-Blackfan. Anemia. Transfusion.

\section{INTRODUÇÃO}

A Síndrome de Blackfan-Diamond (SBD) é uma anemia congênita rara, com uma incidência estimada de $5-10 / 10^{6}$ nascidos vivos. (VLACHOS et al, 2013; DA 
COSTA, NARLA \& MOHANDAS, 2018) A síndrome é hereditária em 40-45\% dos casos por herança autossômica dominante e os 55-60\% restantes são esporádicos. A herança autossômica recessiva foi relatada com menor frequência. (ENDIDAYE, MELKU \& ENAWGAW, 2019)

As manifestações surgem no início da vida, geralmente antes dos 6 meses de idade (média entre os 2-4 meses). (BRAVO \& RODRÍGUEZ, 2000; ALTER \& YOUNG, 1999) A palidez como consequência da anemia é uma das principais alterações, associada com evolução estaturo-ponderal comprometida. (DA COSTA et al, 2001)

O diagnóstico é realizado nos primeiros meses de vida em $90 \%$ dos casos. (DA COSTA, NARLA \& MOHANDAS, 2018) A avaliação é iniciada após a identificação na infância de anemia hipoproliferativa macrocítica, eventualmente normocítica, com hipoplasia eritróide na medula óssea (precursores eritróides correspondendo a menos de $5 \%$ da celularidade).(DA COSTA et al, 2001)

O tratamento de primeira linha da SBD é feito com corticosteroides, embora seu mecanismo de ação ainda seja desconhecido, com resposta observada em $80 \%$ dos pacientes. O programa transfusional de longo prazo é iniciado nos pacientes não responsivos à corticoterapia. A quelação de ferro é necessária para os pacientes em programa transfusional. O transplante de medula óssea alogênico, na presença de um doador HLA-compatível, é o único tratamento curativo da SBD, mas sua indicação continua controversa. (VLACHOS et al ,2008)

O trabalho tem como objetivo relatar o caso de um paciente do sexo masculino de 11 anos de idade, portador desta rara síndrome e refratário ao tratamento com corticoterapia.

\section{RELATO DO CASO}

Paciente do sexo masculino, 11 anos de idade, natural e residente em Toledo, Paraná.

Paciente nasceu com 2,900 kg e é o único filho de seus pais. Mãe relatou que não houve intercorrências durante gestação e parto.

O paciente se apresentou pálido e taquicárdico nos primeiros dias de vida. Ao exame laboratorial, hemograma demonstrou anemia grave, com hemoglobina $(\mathrm{Hb})$ de $2,8 \mathrm{~g} / \mathrm{dL}$, hematócrito de $8 \%$ e volume corpuscular médio (VCM) de 78,43fL. Paciente recebeu transfusão sanguínea devido ao quadro apresentado. Pais negaram histórico de anemia na família.

O exame de aspirado de medula óssea foi realizado aos 3 meses de idade, com resultado hipocelular na linhagem eritroide, sugestivo de síndrome de BlackfanDiamond. O manejo com prednisona foi iniciado e o paciente passou a receber transfusões sanguíneas a cada 30 dias.

O paciente foi encaminhado ao serviço de referência aos 7 meses de idade para avaliação de transplante de medula óssea, mas não foi encontrado doador HLAcompatível e o tratamento com prednisona foi continuado.

O paciente foi reencaminhado para avaliação de transplante de medula óssea aos 2 anos de idade, pois o tratamento com prednisona não obteve resultado e foi suspenso devido à refratariedade. Novamente não foi encontrado doador HLAcompatível. O programa de transfusão de concentrado de hemácias continuou a ser realizado a cada 30 dias e a quelação de ferro foi prescrita. Paciente iniciou o uso de deferasirox $250 \mathrm{mg}$.

A dificuldade de ganho ponderal persistiu durante a infância do paciente, que com 8 anos de idade apresentava peso de $19 \mathrm{~kg}$ (considerado muito baixo para a 
idade). A transfusão crônica de concentrado de hemácias resultou em dosagem de ferritina cada vez maior (valores entre 780-2000) e a dosagem da quelação de ferro foi aumentada para $750 \mathrm{mg}$ de deferasirox associado à desferroxamina.

Os valores de hemoglobina estavam cada vez mais baixos (em torno de $4 \mathrm{~g} / \mathrm{dL}$ ) e, aos 9 anos de idade, as transfusões passaram a ser realizadas a cada 21 dias, pois o intervalo de um mês não era mais tolerado.

A corticoterapia com prednisona foi reavaliada com 10 anos de idade, porém após quatro meses foi suspensa novamente devido à falta de resposta.

Atualmente paciente possui 11 anos de idade e permanece com evolução estaturo-ponderal abaixo do percentil. Paciente continua no programa transfusional associado à quelação de ferro enquanto aguarda doador HLA-compatível para realização de transplante de medula óssea.

\section{DISCUSSÃO}

A síndrome de Blackfan-Diamond é uma doença hematológica rara e as principais alterações estão relacionadas à anemia. A dificuldade no ganho ponderal e malformações, principalmente deformidades craniofaciais e dos membros superiores, são comuns. Baixo peso ao nascer e insuficiência renal e cardíaca também podem estar presentes. (VILAN et al, 2008; IWAFUCHI, 2018) Baixo peso e retardo do crescimento foram documentados em $28,4 \%$ e $22,1 \%$ dos pacientes, respectivamente. A baixa estatura é componente das malformações da síndrome, muitas vezes exacerbada pela longa terapia com esteroides. (DA COSTA et al, 2001)

O diagnóstico é sugerido na presença de anemia hipoproliferativa grave, com redução na medula óssea de precursores eritróides. (ALTER \& YOUNG, 1999) A anemia é moderada ou grave: a hemoglobina na apresentação varia entre $1,5 \mathrm{~g} / \mathrm{dL}$ e $12,4 \mathrm{~g} / \mathrm{dL}$. A contagem de leucócitos e plaquetas geralmente é normal: a neutropenia é identificada em $25 \%$ dos pacientes e a trombocitopenia ou trombocitose pode estar presente no diagnóstico. (DA COSTA et al, 2001) Grande parte dos pacientes apresentam hemoglobina fetal ( $\mathrm{HbF}$ ) e adenosina desaminase (eADA) aumentadas. (VLACHOS \& MUIR, 2010)

Mutações ou deleções são identificadas em aproximadamente $70 \%$ dos casos, na maioria das vezes por haploinsuficiência de uma proteína ribossômica: a mutação no gene codificador da proteína RPS19 é a mutação mais prevalente (25\%). Outros genes ribossomais envolvidos são: RPS24, RPS17, RPL54A, RPL5, RPL11, RPS7, RPS10, RPS26, RPL26. (SAKAMOTO \& NARLA, 2017; DA COSTA, TCHERNIA \& LEBLANC, 2008) Mutações envolvendo o fator de transcrição eritróide GATA1 foram encontradas em alguns casos. (ENGIDAYE, MELKU \& ENAWGAW, 2019)

O paciente avaliado apresentou anemia grave nos primeiros dias de vida, com hemoglobina de 2,8 g/dL e dificuldade no ganho de peso. A suspeita diagnóstica de Blackfan-Diamond foi confirmada pela hipocelularidade acentuada na série eritróide medular. Testes genéticos não foram realizados.

A probabilidade de aborto de um feto portador da doença, bem como a probabilidade de parto prematuro, é maior do que na população geral. Manifestações pré-natais importantes como a hidropsia fetal são raramente encontradas. (DA COSTA et al, 2001) Parto prematuro e manifestações pré-natais não foram observadas no caso descrito.

A corticoterapia, na dose inicial de $2 \mathrm{mg} / \mathrm{kg} / \mathrm{dia}$, é a primeira opção terapêutica e é efetiva em $70-80 \%$ dos casos relatados. A incidência de complicações é reduzida e o prognóstico e a qualidade de vida são melhores nos pacientes responsivos. 
(WILLIG, GAZDA \& SIEFF, 2000) A interleucina 3 (IL3), a ciclosporina (isolada ou em associação com corticoesteroides), a metoclopramida e o ácido valpróico foram testados e obtiveram respostas em um número mínimo de pacientes, não sendo considerados terapia padrão da síndrome. (DA COSTA et al, 2008) O transplante alogênico de medula óssea é o único método de cura para a doença na presença de um doador HLA-compatível. (VLACHOS et al, 2008)

No caso relatado o paciente não respondeu à corticoterapia e deu continuidade ao tratamento apenas com o programa de transfusão sanguínea a cada 21 dias somado à quelação de ferro. O paciente não realizou o transplante devido à ausência de um doador HLA-compatível. Os demais tratamentos alternativos não foram realizados.

A sobrevida global dos pacientes é de $75 \%$ até os 40 anos de idade e a mediana da sobrevida global é de 58 anos. (DA COSTA, NARLA \& MOHANDAS, 2018) Complicações do tratamento, infecções e sobrecarga de ferro são as principais causas de morte dos portadores da síndrome. (IWAFUCHI, 2018)

A sobrecarga de ferro é o principal problema relacionado à terapia transfusional, pois pode causar hemossiderose cardíaca, diabetes mellitus, cirrose hepática, hipoparatireoidismo, hipotireoidismo e puberdade tardia. A concentração hepática de ferro obtida por biópsia é o melhor exame para a identificação da sobrecarga de ferro. A dosagem da ferritina sérica é o exame mais utilizado, por não ser invasivo e possuir baixo custo, entretanto pode estar elevada na presença de processos inflamatórios. (VLACHOS \& MUIR, 2010)

O paciente do caso descrito é dependente de transfusão sanguínea a cada 21 dias e faz uso dos quelantes desferroxamina e deferasirox, porém apresentou elevação crônica da ferritina. A sobrecarga de ferro é uma patologia que deve ser considerada no caso, sobretudo a longo prazo, mesmo em uso de terapia de quelação.

\section{CONCLUSÕES}

O paciente, com anemia grave desde o nascimento, diagnosticado como portador da síndrome de Blackfan-Diamond por aspirado de medula óssea, não apresentou resposta ao tratamento de primeira linha com corticosteróides e continua dependente de transfusão sanguínea periódica e terapia de quelação de ferro. Seu estado geral é bom, apresenta desenvolvimento estaturo-ponderal comprometido e mantém, apesar da terapia quelante, estoques corporais de ferro aumentados.

\section{REFERÊNCIAS}

ALTER B.; YOUNG, N. The bone marrow failure syndromes. Clinics in Laboratory Medicine, v. 10, p. 113-134, 1999. https://doi.org/10.1016/S0272-2712(18)30131-8

BRAVO L. M.; RODRÍGUEZ Z. N. Anemia de Diamond-Blackfan: Experiencia clínica en 20 pacientes (1968-1998). Revista chilena de pediatria, v. 71, n. 3, 2000. http://dx.doi.org/10.4067/S0370-41062000000300003

DA COSTA L.; WILLIG TN.; FIXLER J.; MOHANDS N.; TCHERNIA G. DiamondBlackfan anemia.Curr Opin Pediatr, v. 13, p.10-15. 2001, https://doi.org/10.1097/00008480-200102000-00002 
DA COSTA L.; NARLA A.; MOHANDAS N. An update on the pathogenesis and diagnosis of Diamond-Blackfan anemia. F1000Research, v. 7, p. 1350, 2018. https://doi.org/10.12688/f1000research.15542.1

DA COSTA L.; TCHERNIA G.; LEBLANC T. Diamond-Blackfan anaemia. EBMT, p. 142-163, 2008.

ENGIDAYE G.; MELKU M.; ENAWGAW, B. Diamond Blackfan Anemia: genetics pathogenesis, diagnosis and treatment. eJIFCC, v. 30, n. 1, p. 67-81, 2019. PMID: 30881276; PMCID: PMC6416817

IWAFUCHI H. The histopathology of bone marrow failure in children. Journal of Clinical and Experimental Hematopathology, v. 58, n. 2, p. 68-86, 2018. https://doi.org/10.3960/is/rt.18018

SAKAMOTO K.; NARLA A. Perspective on Diamond-Blackfan anemia: lessons from a rare congenital bone marrow failure syndrome. Leukemia, v. 32, n. 2, p. 249-251, 2018

https://doi.org/10.1038/leu.2017.314

VILAN A.; CUNHA J.; BRANDÃO C.; CLETO E. Anemia de Diamond-Blackfan. Acta Pediatr Port, v. 39, n. 2, p. 72-74, 2008. https://doi.org/10.25754/pjp.2008.4558

VLACHOS A, et al. Diagnosing and treating Diamond Blackfan anaemia: results of an international clinical consensus conference. British Journal of Haematology, v. 142, n. 6, p. 859-876, 2008. https://doi.org10.1111/j.1365-2141.2008.07269.x

VLACHOS A.; MUIR E. How I treat Diamond-Blackfan anemia. Blood, v. 116, n. 19, p. 3715-3723, 2010 https://doi.org/10.1182/blood-2010-02-251090

VLACHOS A.; DAHL N.; DIANZANI I.; LIPTON J. Clinical utility gene card for: Diamond - Blackfan Anemia - update 2013. European Journal of Human Genetics, v. 21, n. 10, p. 1187, 2013. https://doi.org/10.1038/ejhg.2010.247

VLACHOS A, et al. Incidence of neoplasia in Diamond Blackfan anemia: a report from the Diamond Blackfan Anemia Registry. Blood, v. 119, n. 16, p. 3815-3819, 2012. https://doi.org/10.1182/blood-2011-08-375972

WILLIG T.; GAZDA H.; SIEFF C. Diamond-Blackfan anemia. Current Opinion in Hematology, v. 7, n. 2, p. 85-94, 2000. https://doi.org/10.1097/00062752200003000-00003 